\title{
Multiple-choice questions in gastro-enterology and hepatology
}

\author{
M C Bateson, R Holden
}

Are the statements listed beneath each question true or false? Answers are given overleaf.

\section{QUESTION 1}

Which cancers are becoming commoner in the United Kingdom:
A Pancreas
B Liver
C Stomach
D Duodenum
E Gallbladder

\section{QUESTION 2}

A patient with Crohn's disease presents with an exacerbation of diarrhoea. He is toxic with a slight fever, a right iliac fossa mass and right sided loin pain. There are no features of intestinal obstruction. Which of the following is true:

A Ultrasound examination is helpful

B Treatment with steroids should be started

C Urgent laparotomy is required

D Metronidazole should be added to the treatment

E Azathioprine is rapidly effective

\section{QUESTION 3}

Characteristic abnormalities of small bowel are found on peroral biopsy in which of the following conditions:
A Coeliac disease
B Alactasia
C Amyloidosis
D Tropical sprue
E Chronic radiation enteritis

\section{QUESTION 4}

Acute appendicitis:

A Is diagnosed by pain starting in the right iliac fossa associated with low-grade fever and leucocytosis

B Is commoner in young people

C Leads to the commonest abdominal operation

General Hospital, Bishop Aukland, County Durham DL14 6AD, UK M C Bateson

Monklands Hospital, Airdrie, UK

R Holden

Accepted 16 December 1997
D Is linked to severe complications in a minority irrespective of age

E Is associated with inflammatory bowel disease

\section{QUESTION 5}

Which of the following drugs reduce portal pressure in patients with portal hypertension:
A Nitrates
B Aspirin
C Beta-blockers
D Spironolactone
E Chenodeoxycholic acid

\section{QUESTION 6}

A 65-year-old man is admitted as an emergency with severe epigastric pain. Next day, 'liver function tests' show a bilirubin of $38 \mu \mathrm{mol} / \mathrm{l}$ (normal < 20), alkaline phosphatase 350 IU/1 $(<100)$, $\gamma$-glutamyl transferase 42 IU/l $(<50)$, alanine transaminase 87 IU/I $(n<60)$, albumin $39 \mathrm{~g} / \mathbf{l}(>35)$, and globulin $22 \mathrm{~g} / \mathbf{l}(<30)$. A haemogram was normal with less than $1 \%$ reticulocytes:

A This could be biliary colic

B Acute hepatitis is a likely diagnosis

C The bilirubin level may reflect Gilbert's syndrome

D Serum calcium should be checked

E Benign intrahepatic cholestasis is a differential diagnosis

\section{QUESTION 7}

The finding of pigmentation in the colonic mucosa-melanosis coli-in a patient with diarrhoea suggests which of the following diagnoses:
A Addison's disease
B Ulcerative colitis
C Bile salt malabsorption
D Haemochromatosis
E Laxative abuse

\section{QUESTION 8}

In a patient presenting with abdominal pain, weight loss and diarrhoea, which of the following investigations suggest pancreatic insufficiency:

A Pancreatic calcification on plain X-ray of abdomen

B Serum albumin $23 \mathrm{~g} / 1$

C Low serum folate

D Abnormal fluorescein dilaurate test

E Hypocalcaemia with raised serum alkaline phosphatase 
Answers

\section{QUESTION 1}

A True

B True

C False

D False

E False

Cancer of the pancreas is commoner than primary liver cancer but both are increasing in frequency. Overall gastric cancer frequency has fallen for many years, though this relates only to antral and pyloric tumours rather than proximal ones. The rate of gallbladder cancer is probably not changing, but diagnosis will clearly depend on the rate of cholecystectomy. Duodenal carcinoma remains very rare.

\section{QUESTION 2}

A True

B True

C False

D True

E False

Ultrasound examination will help in deciding if there is abscess present in addition to an inflammatory mass due to adherent loops of inflamed bowel. Hydronephrosis may occur in these circumstances and will be seen on ultrasound. Treatment with steroids and metronidazole are appropriate. Azathioprine has no immediate effect in the treatment of acute exacerbations of Crohn's disease because of its slow onset of action, but long-term treatment may usefully start in an acute attack. Surgery is not indicated urgently.

\section{QUESTION 3}

A True

B False

C True

D True

E False

There is no morphological abnormality in alactasia. Chronic radiation enteritis produces a submucosal endarteritic lesion and will not usually be detected in the more superficial specimens obtained by peroral biopsy.

\section{QUESTION 4}

A False

B True

C True

D True

E True

Appendicitis pain begins centrally. The peak frequency of appendicectomy is in teenage and the $20 \mathrm{~s}$, though perforation, peritonitis and appendix abscess are distributed throughout the age range. A history of appendicectomy is commoner in Crohn's disease, which might be explained by confusion of diagnosis. However, it is also commoner in ulcerative colitis where the association is harder to explain, but might have an immune basis.

\section{QUESTION 5}

A True

B False

C True

D True

E False

Beta-blockers and nitrates are important agents for primary and secondary prophylaxis of bleeding from varices. Nitrates used in conjunction with intravenous terlipressin in acute bleeding increase efficacy and reduce peripheral side-effects. The clinical utility of the effect with spironolactone is uncertain.

\section{QUESTION 6}
A False
B False
C True
D True
E False

Although serum biochemistry tests are conveniently grouped under 'liver function tests' they may not reflect liver disease. Markedly raised alkaline phosphatase with normal $\gamma$-glutamyl transferase suggests a non-hepatic problem and Paget's disease is a common cause. Similarly, in biliary and pancreatic disease these two enzymes tend to rise together. Bone disease may lead to hypercalcaemia, particularly if the patient is immobilised. The pattern of enzymes observed is unlike acute hepatitis. The electrocardiogram showed a inferior myocardial infarction which had precipitated unconjugated hyperbilirubinaemia in a patient with Gilbert's syndrome and a background of Paget's disease.

\section{QUESTION 7}
A False
B False
C False
D False
E True

The finding of melanosis coli, a distinct blackspeckled pigmentation of the colonic mucosa, at sigmoidoscopy indicates regular use of anthroquinone laxatives.

\section{QUESTION 8}
A True
B False
C False
D True
E False

Pancreatic calcification, when present, is diagnostic of chronic pancreatitis and indicates a disease duration of greater than 10 years. In the fluorescein dilaurate test pancreatic lipase splits fluorescein from the dilauric acid, and the fluorescein is absorbed and excreted in the urine where it is measured. Other causes of malabsorption are more likely to produce hypoproteinamia and vitamin deficiency. 